\title{
CESTODES TÉTRAPHYLLIDES NOUVEAUX OU PEU CONNUS DE DASYATIS PASTINACA (L.)
}

\author{
Par Louis EUZET
}

La Tère ou Pastenague (Dasyatis pastinaca (L.) Sélacien) est parasitée par un certain nombre de Cestodes Tétraphyllides. Joyeux et BaEr, dans la Faune de France, ne citent pas moins de 10 espèces différentes chez cette Raie. Bien que, dans cette liste, plusieurs parasites demandent à être revus, nous pensons qu'elle devra s'allonger encore. En effet, chez Dasyatis pastinaca (L.) de nos côtes doivent exister certaines espèces de Tétraphyllides décrites chez des Dasyatidæ des côtes atlantiques nord-américaines.

Parmi les parasites cités, quelques-uns ne sont connus que par les descriptions anciennes. En outre, comme nous le verrons plus loin, les synonymies établies, en particulier par Southwell, sont venues compliquer les choses à un point que certains genres, tel Echeneibothrium, sont devenus un fouillis inextricable.

La présente note, après celle de Dollfus 1926 et BAER 1948, doit aider à une connaissance plus complète des parasites de Dasyatis pastinaca (L.). Elle a pour but :

$1^{\circ}$ de préciser la morphologie et l'anatomie de Rhinebothrium tumidulum. Cette espèce, décrite en 1819 par Rudolphi sous le nom de Bothriocephalus tumidulus chez Dasyatis pastinaca, ne semble pas avoir été revue depuis ;

$2^{\circ}$ de donner quelques précisions sur Rhinebothrium walga (SHIPley et HoRnell 1906);

$3^{\circ}$ de décrire Rhabdotobothrium dollfusi, pour lequel nous serons amené à créer une espèce nouvelle dans un genre nouveau.

\section{1) RHINEBOTHRIUM TUMIDULUM (RUDOLPHI 1819)}

Syn. Bothriocephalus tumidulus Rud. 1819.

Bothriocephalus tumidulus BREMSER 1819.

Bothriocephalus echeneis LeUcKART 1819 (pro parte).

Ann. de PARasitologie, T, XXVIII, $\mathrm{N}^{\circ}$ 5-6. - 1953, 
Bremser a envoyé à Rudolphi un fragment de «Bothriocéphale » de 5 lignes de long $(11 \mathrm{~mm}$.), provenant de Dasyatis pastinaca (L.). Ce ver a servi à Rudolphi de type pour l'espèce qu'il a désignée en 1819 sous le nom de Bothriocephalus tumidulus et qu'il définissait ainsi : « Bothriis utrinque duobus ovalibus crassiusculis linea divisis, transversim striatis, collo brevissimo - articulis primis angistissimis elongatis in sequentibus subquadratis. Hab. in intestinis Raja pastinacea L. 》

Pendant l'année 1824, parut l'Atlas de Bremser où était dessiné le Bothriocephalus tumidulus RudolphI. Il ne fait aucun doute que le ver dessiné par Bremser soit celui qu'il avait envoyé à Rudolphi, car figure et description concordent.

En 1820, Leuckart faisait paraitre une "Monographie du genre Bothriocéphale ». Cet auteur avait eu à sa disposition les collections de Bremser, du Musée de Vienne, et nous retrouvons, sous le nom de Bothriocephalus echeneis, un ver qui ressemble, tout au moins en partie, à l'espèce de Rudolphi telle que l'a dessinée Bremser.

Ce sont là les seuls renseignements précis que l'on ait sur cette espèce, dont plusieurs auteurs ont parlé par la suite. VAN BENEDEN (1850) le cite et, d'après les dessins de BrEmser, le déclare différent de son Echeneibothrium minimum et de son Echeneibothrium variabile.

DiEsING, en 1863, cite et décrit un Echeneibothrium tumidulum d'après VAN BENEDEN?

Carus, en 1884, groupe sous le nom d'Echeneibothrium tumidulum (Rud. 1819) les espèces de VAN BENEden.

Southwell suit cette voie et place sept espèces en synonymie avec celle de Rudolphi. Ce sont : Echeneibothrium variabile VAN BENEDEN 1850 ; E. affine OLSSON 1867 ; E. gracile ZCHOKKE 1888 ; Tritaphros retzii LoENNBERG 1889 ; Rhinebothrium longicolle LINTON $1890 ;$ E. ceylonicum SHIPLEY et HorNell $1906 ; R$. maccallumi Linton $1924 ;$ E. austrinum Linton 1924.

SouthWELl, voulant ici encore simplifier la nomenclature en réduisant le nombre d'espèces, n'a fait que la compliquer un peu plus.

Malheureusement, après lui, Joyeux et BAER citent Echeneibothrium tumidulum Rud 1819 dans la Faune de France, Cestodes, 1936.

WARDLE et MAC Leod écrivent en 1952 dans Zoology of tapeworms, toujours à propos de ce ver : "Etabli d'après le Bothriocephalus tumidulus Rudolphr 1819 de Raja pastinaca. Localité inconnue. Extrêmement variable dans sa forme. Ce nom spécifique est applicable à toutes les formes d'Echeneibothrium, sans myzorhynchus, 
qui ont une double rangée de loculi à chaque bothridie ». Autrement dit, ce nom spécifique peut s'appliquer à toutes les espèces du genre Rhinebothrium, tel que l'a défini Linton en 1890.

Nous avons retrouvé un Cestode qui correspond à la description de Rudolphi et au dessin de Bremser. Nous assimilons cette espèce au Bothriocephalus tumidulus. Le matériel de Rudolphi, du Musée de Berlin, que nous avons examiné, est inutilisable, aucun détail caractéristique n'étant visible ; nous donnons une nouvelle description de ce parasite avec quelques précisions sur sa morphologie et son anatomie.

Hôte : Dasyatis pastinaca (L.), Concarneau (Finistère), R. LegenDre, leg. (Coll. R.-Ph. Dollfus).

Ces parasites montés et colorés mesurent de 10 à $15 \mathrm{~mm}$. de long avec une largeur maxima de $700 \mu$. Nous avons compté de 80 à à 100 segments, tout d'abord comme un simple trait transverse, puis s'allongeant très lentement pour devenir dans les derniers à peine plus longs que larges. Les segments sont acraspèdes. Les strobiles que nous avons examinés sont tous sexués, mais aucun proglottis n'est gravide. Les derniers segments mesurent en moyenne $800 \mu$ de long sur $700 \mu$ de large.

Anatomie externe : Le scolex (fig. 1) est essentiellement formé de quatre grandes bothridies brièvement pédonculées. Ces bothridies sont groupées par deux (une paire dorsale et une paire ventrale). Le scolex est, de ce fait, légèrement aplati dorso-ventralement. La partie postérieure de la bothridie se replie en bénitier, lui donnant un aspect particulier qu'ont bien dessiné Bremser et Leuckart. Sur chaque bothridie, on remarque un septum médian longitudinal très saillant. De part et d'autre de cette crête, nous avons compté 10 septums musculaires transverses, qui divisent la bothridie en 22 loculi. A la partie antérieure, on distingue un tout petit loculus apical en secteur. Nous donnons ces chiffres avec un point de doute, le décompte des septums est en effet très malaisé dans la partie postérieure repliée en bénitier. Chaque bothridie mesure de $1 \mathrm{~mm}$. à $1,2 \mathrm{~mm}$. de long sur $600 \mu$ à $700 \mu$ de large. Sur nos préparations, il n'y a pas de myzorhynchus visible. Le pédoncule céphalique est absent. Le cou est court. Cette première partie du strobile n'a pas chez nos exemplaires l'allure à gros anneaux que Bremser et LEUCKaRT ont dessiné. Est-ce un effet particulier de contraction chez les individus fixés?

Le pore génital alterne irrégulièrement. Il s'ouvre au milieu du bord latéral du segment. 


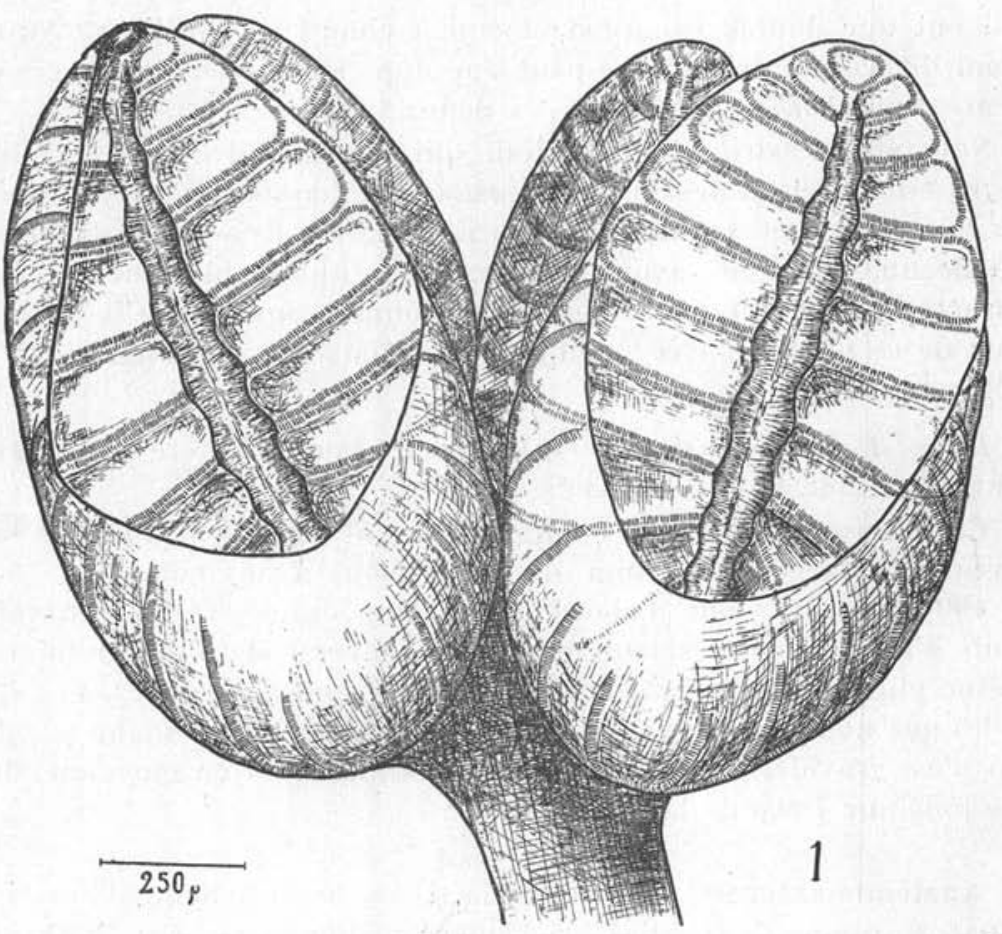

Fig. 1. - Rhinebothrium tumidulum (Ruv. 1819). Préparation totale d'un scolex

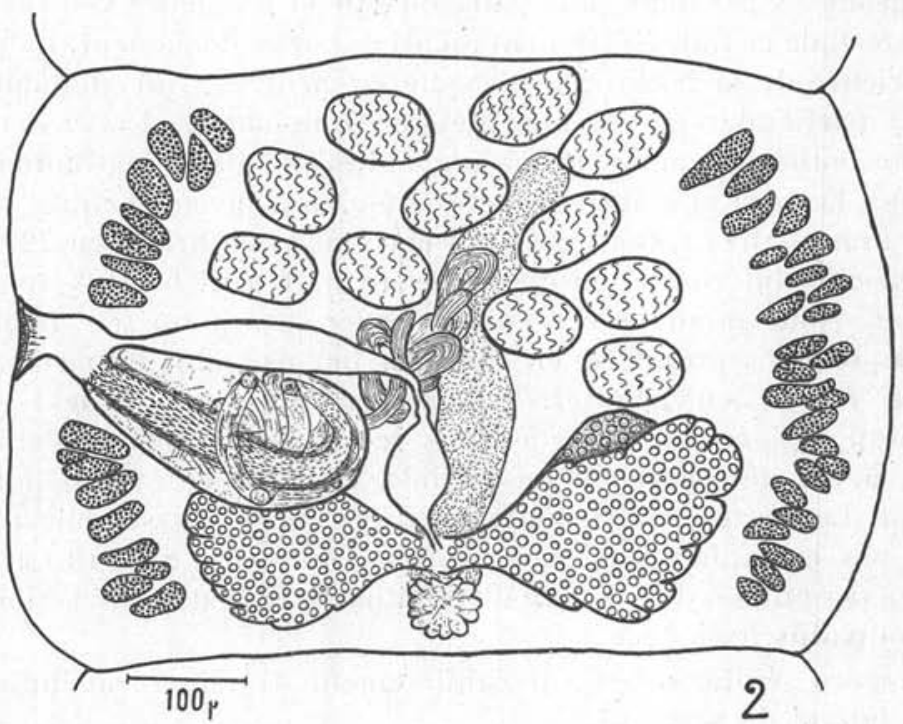

FIG. 2. - Rhinebothrium tumidulum (RrD. 1819). Proglottis sexué 
Anatomie interne (fig. 2), - Appareil mâle: Les testicules sont situés à la partie antérieure du segment. Leur nombre varie de 10 à 12. Le vas deferens apparaît dans les derniers segments du strobile. Il forme un peloton entre les groupes de testicules et à l'extrémité antiporale de la poche du cirre. Celle-ci est grande $(225 \mu$ sur $75 \mu$ ). Allongée, elle atteint le milieu du segment. Elle contient un pénis armé d'épines que nous n'avons pas vu évaginé.

Appareil femelle : Le vagin débouche antérieurement à la poche du cirre. Il se dirige d'abord horizontalement vers le milieu de l'anneau par un conduit élargi de $50 \mu$ de diamètre. Au milieu du segment, il se rétrécit en un étroit canal qui fait un coude. Le vagin descend alors vers l'ovaire en s'élargissant et forme un réceptacle séminal de 50 à $60 \mu$ de diamètre. L'ovaire tétralobé s'étend à la partie postérieure de l'anneau. D'abord très aplati, il prend de l'importance à mesure que les segments s'allongent.

Les vitellogènes en gros follicules forment deux doubles bandes latérales importantes non interrompues au niveau de la poche du cirre.

L'utérus dans les anneaux les plus mûrs que nous ayons examinés se présente comme une bande sinueuse à la partie médiane du segment. Nous n'avons pas eu de proglottis gravide.

Discussion : Ce Cestode, d'après la description que nous venons de donner, entre dans le genre Rhinebothrium Linton 1890, emended BAER 1948.

Parmi les espèces de ce genre ainsi défini, Rhinebothrium flexile Linton 1889 se rapproche de $R$. tumidulum par le nombre de testicules. Mais l'allure et la taille du scolex, le nombre de loculi généralement plus élevé, le strobila mince avec des segments rapidement plus longs que larges l'en différencient nettement.

Rhinebothrium maccallumi Linton 1924 ressemble comme allure du scolex à Rhinebothrium tumidulum, mais le nombre de loculi et surtout de testicules (4-5) l'en sépare. Nous avons pu à ce sujet nous en convaincre en examinant la préparation originale de Linton (U.S.N., Mus. Helm., Coll. $\mathrm{n}^{\circ}$ 7.665).

\section{2) RHINEBOTHRIUM WALGA (Shipley et Hornell 1906)}

Nous avons récolté cette espèce dans la valvule spirale de Dasyatis pastinaca à Sète et l'avons retrouvée toujours en petite quantité parmi les Cestodes de cette Raie de la Collection R.-Ph. Dollfus. Les Dasyatis de cette collection provenaient de Concarneau (Finistère) R. Legendre Lég. ; Alexandrette (Syrie) W. Besnard Lég. et Agigea (Roumanie) Bacesco leg. 
Nous avons cru au premier abord avoir là l'Echeneibothrium minimum P. J. VAN BENEDEN 1850. La disposition et le nombre de testicules sont en effet semblables, mais l'anatomie en est différente. Nous avons par ailleurs retrouvé parmi les parasites de Dasyatis marmorata (D. pastinaca, var. marmorata) STEINDACHNER, de Gorée (Sénégal), Collection I.F.A.N., un Cestode qui correspond à l'espèce de VAN BEnEden.

Le ver qui nous occupe ressemble beaucoup à l'espèce que SHIPLEY et Hornell ont décrite en 1906 sous le nom d'Echeneibothrium walga. Nous n'avons pu examiner le matériel-type de ces auteurs. Malgré cela, nous assimilons ce Cestode à l'espèce de Trygon walga.

Nous en donnons une briève description, qui complétera celle donnée par Shipley et Hornell.

Anatomie externe : Le scolex (fig. 3) est très variable dans sa forme et son aspect. Il est formé par quatre bothridies, chacune portée par un long pédoncule musculeux rétractile, qui peut s'infléchir en tout sens. Les bothridies elles-mêmes sont très mobiles. Elles sont divisées par un septum longitudinal en deux rangées, chaque rangée elle-même divisée par des septums transverses en 20 loculi. Elles possèdent en outre un loculus impair en secteur à chaque extrémité. Les bothridies étalées mesurent environ $600 \mu$ de long sur 100 à $150 \mu$ de large. Leur dos, ainsi que la surface des pédoncules sont recouverts de petites épines.

Il n'y a pas de myzorhynchus.

Au scolex fait suite un pédoncule céphalique musculeux de $200 \mu$ de long. Ce pédoncule est recouvert de petites épines dirigées vers l'arrière. Les pores sexuels latéraux alternent irrégulièrement, et débouchent dans un atrium génital généralement bien marqué. Ce pore est situé au milieu du segment, au tiers antérieur dans les proglottis les plus allongés.

Anatomie interne (fig. 4). - Appareil mâle: Les testicules en petit nombre, 4 à 6 , sont situés à la partie antérieure du segment. Le vas deferens est très développé dans les derniers segments du strobile et forme des circonvolutions dans toute la partie antérieure, où il remplace les testicules qui ont dans ces anneaux presque disparu. La poche du cirre globuleuse, petite, mesure $90 \mu$ sur $60 \mu$. Elle contient un pénis armé de petites épines. Ce cirre évaginé présente une dilatation à la base. Il mesure de $175 \mu$ à $200 \mu$ de long. Le revêtement d'épines paraît plus dense sur la dilatation basale.

Appareil femelle : Le vagin débouche dans l'atrium génital, antérieurement à la poche du cirre. Sa paroi est musculaire ; son trajet 
très simple. Il forme un coude en contournant la poche du cirre et descend ensuite vers l'ovaire. Celui-ci est tétralobé, très allongé dans les derniers segments. Les vitellogènes forment deux groupes latéraux en deux bandes (une dorsale et une ventrale). Elles sont composées de petits follicules très rapprochés.

L'utérus se présente dans les segments mûrs comme un tube médian. Nous n'avons pas eu de proglottis gravides.

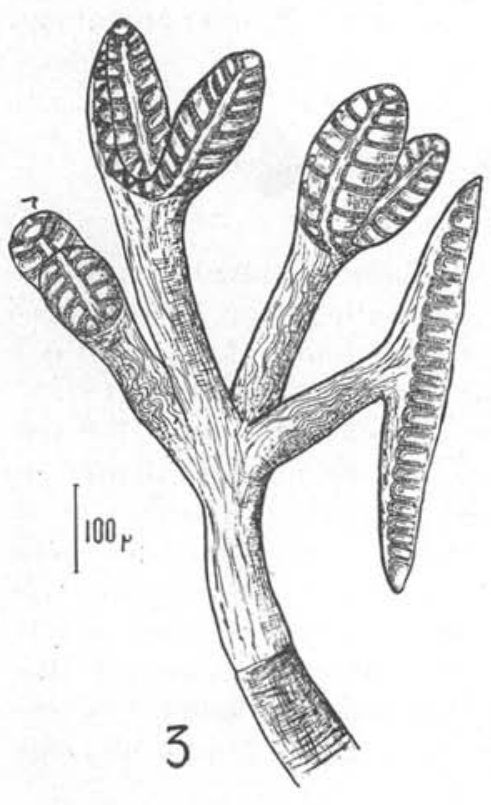

Fig. 3. - Rhinebothrium walga $\left(\mathrm{S}_{\mathrm{H} 1-}\right.$ PLEY et Hounel.L 1906). Préparation totale d'un scolex.

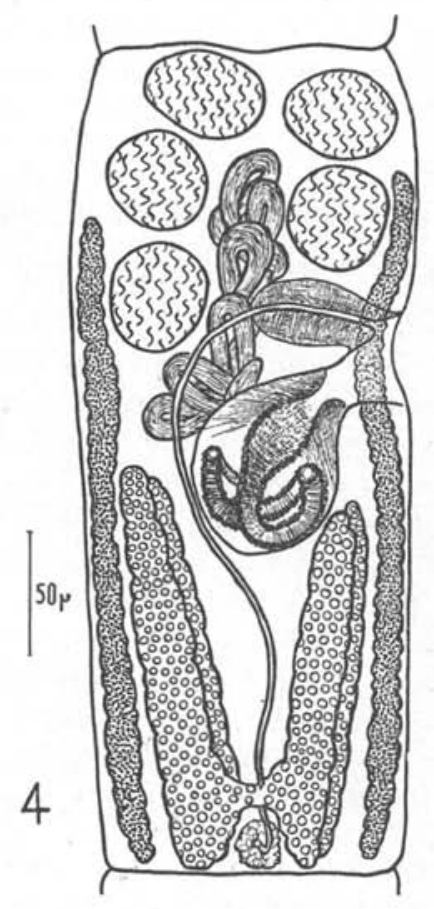

Fig. 4. - Rhinebothrium walga (SHIr.LEY et HonNell 1906). Segment adulte.

Discussion: Les vers que nous avons examinés sont semblables par la taille, l'allure du scolex, le nombre de testicules à l'espèce de ShIPLEY et Hornell, décrite en 1906 sous le nom d'Echeneibothrium walga.

L'absence de myzorhynchus nous permet de classer ce Cestode dans le genre Rhinebothrium Linton 1890 emended BaER 1948. Ce ver doit done se nommer Rhinebothrium walga (SHIPLEY et HoRNELL 1906). 
Rhinebothrium walga ressemble à Rhinebothrium flexile Linton 1890 par l'allure du scolex, mais s'en sépare par le nombre de testicules, généralement plus élevé dans l'espèce de Linton. Il se rapproche de Rhinebothrium maccallumi Linton 1924 par le nombre réduit de testicules, mais s'en sépare par la taille et surtout l'allure du scolex qui est toute différente.

Le ver décrit en 1934 par L. Leon Borcea sous le nom d'Echeneibothrium minimum, hôte : Dasyatis pastinaca (L.), Agigea (Roumanie), doit correspondre à Rhinebothrium walga. Nous ne pouvons cependant l'affirmer avec certitude, cet auteur ne donnant aucun détail sur l'anatomie interne du segment.

\section{3) RHABDOTOBOTHRIUM DOLLFUSI n. g., n. sp.}

Hôte : Dasyatis pastinaca (L.).

Nous avons récolté ce Cestode à Sète, dans la valvule spirale de cette Raie (quatre individus dont un seul adulte). Nous l'avons aussi rencontré parmi les parasites de Dasyatis pastinaca (L.) recueillis à Concarneau (Finistère) par R. Legendre (Coll. R.-Ph. Dollfus).

Le ver entier mesure de 40 à $50 \mathrm{~mm}$. Nous avons compté 150 segments, dont les derniers ont environ $2 \mathrm{~mm}$. de long sur $1 \mathrm{~mm}$. de large. Le strobile est fortement craspédote. Cette disposition ne se voit pas dans les tout premiers segments, tous très serrés, mais elle est bien visible à partir du $30^{\circ}$. Le ver vivant, lorsqu'il est très allongé, prend alors l'allure d'une corde à nœuds. Cestode apolytique, mais nous avons trouvé des segments mûrs à utérus vidé attachés à la chaîne et des segments détachés bourrés d'œufs. Les proglottis libres mesurent jusqu'à $4 \mathrm{~mm}$. de long sur $2 \mathrm{~mm}$. de large.

Anatomie externe : Le scolex (fig. 5) ressemble à celui des Cestodes du genre Rhinebothrium LinTon 1890. Il est essentiellement formé de quatre bothridies pédonculées, très variables dans leur forme et divisées en un certain nombre de loculi par des septums musculaires. Nous avons une charnière longitudinale qui sépare deux rangées latérales, où des septums transverses dessinent 25 loculi. A la partie antérieure, on distingue un loculus apical impair en secteur. Nous avons donc en tout 51 loculi. La bothridie étalée mesure $650 \mu$ sur $250 \mu$. La cuticule du dos des bothridies, ainsi que celle de leur pédoncule sont couvertes d'un tapis serré de petites épines. Il n'y a pas de myzorhynchus. Il n'y a pas de pédoncule céphalique. Le cou est très court. Le strobile est formé, nous l'avons déjà dit, de segments fortement craspédotes. Le pore génital s'ouvre au milieu du bord latéral de l'anneau et alterne irrégulièrement. 
Dans les derniers segments de la chaîne et chez les proglottis détachés, le cirre (volumineux) est très souvent évaginé.

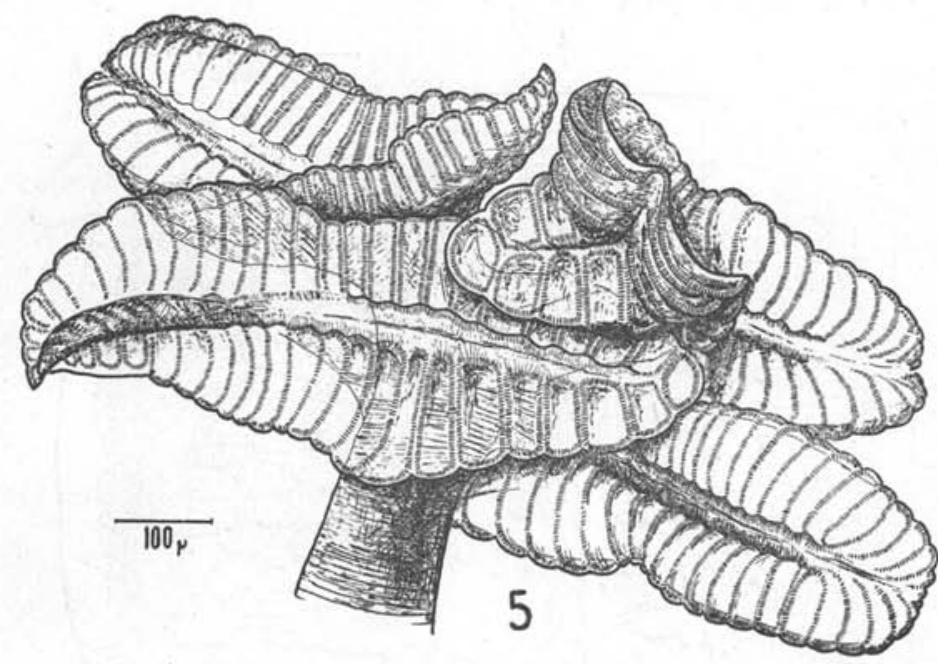

Fig. 5. - Rhabdotobothrium dollfusi n.g.n.sp. Préparation totale d'un scolex

Anatomie interne (fig. 6 et 7). - Appareil mâle: Nous avons compté de 100 à 130 testicules, qui sont répartis en deux champs latéraux. Le champ poral, interrompu au niveau du pore génital, reprend au delà et il y a des testicules (une dizaine) entre l'ovaire et la poche du cirre. Le vas deferens est en général peu développé. Il forme quelques diverticules autour de l'extrémité antiporale de la poche du cirre. Celle-ci est très grande et mesure $1,5 \mathrm{~mm}$. de long sur $650 \mu$ de diamètre. Repliée vers la partie postérieure du proglottis, elle contient un cirre épineux très particulier, qui mérite une description détaillée. Ce pénis est, en effet, formé de deux parties de diamètre différent. Une première partie, très grosse et qui mesure de $1 \mathrm{~mm}$. à $1,5 \mathrm{~mm}$. de long sur $300 \mu$ de diamètre, est bien visible à l'œil nu chez les segments où elle est évaginée. Cette partie, inerme à la base, est, sur les $4 / 5$, recouverte d'épines d'abord très petites et très serrées qui, par la suite, vont en s'accroissant et se raréfiant à mesure que l'on s'approche de l'extrémité. Elles sont formées d'une base arrondie et d'une pointe en épine de rosier. De cette première partie s'évagine la deuxième, beaucoup plus mince $(100 \mu$ de diamètre), mais aussi longue $(1,5 \mathrm{~mm}$.). Comme la première, cette partie est aussi armée d'épines, mais celles-ci sont assez grosses et plus rares. Lorsque les deux parties sont évaginées, l'ensemble prend 
l'aspect d'un fouet, dont la première partie serait le manche et la deuxième la lanière. Notons que la deuxième partie est bien moins souvent évaginée que la première.

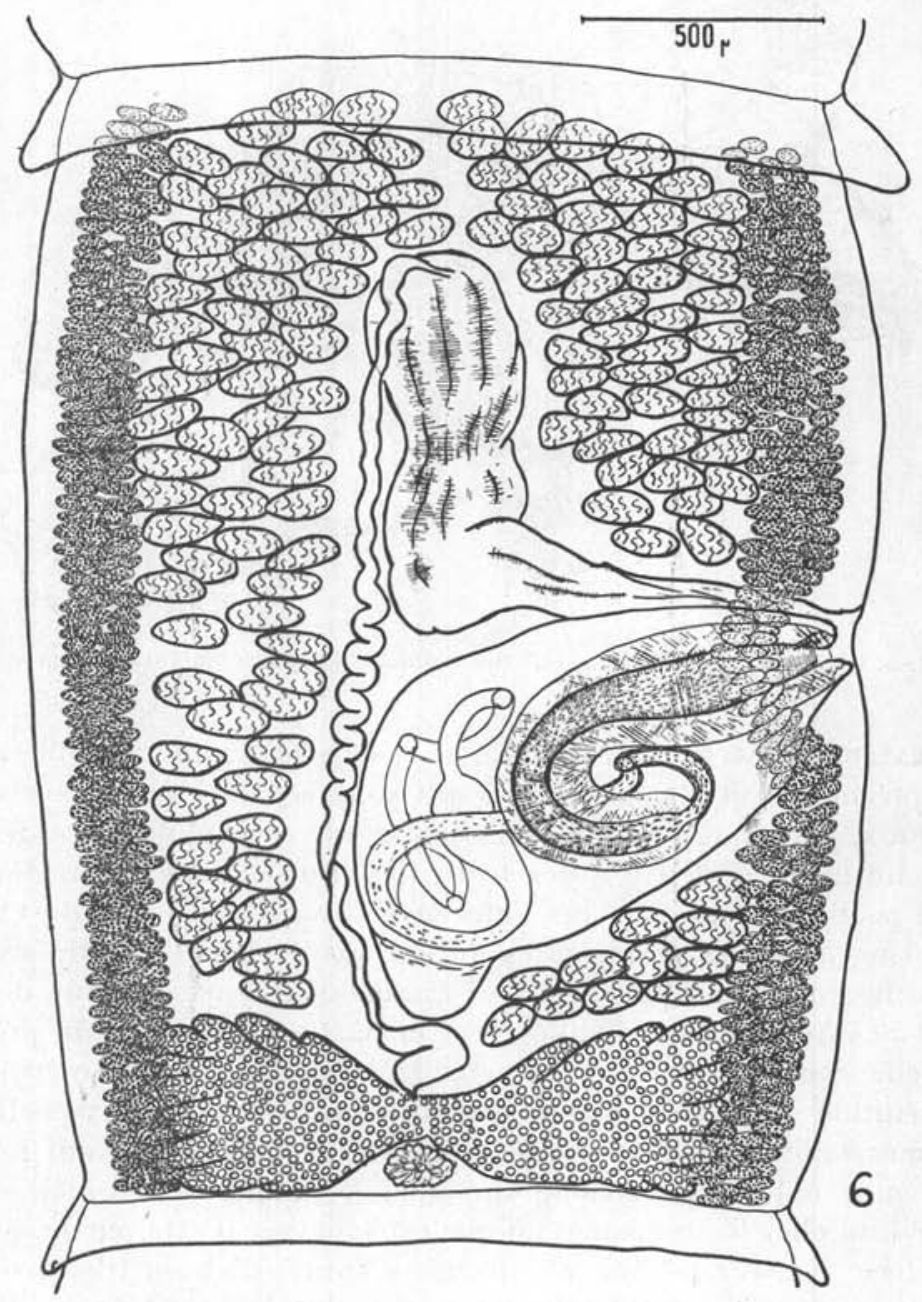

FIG. 6. - Rhabdotobothrium dollfusi n.g.n.sp. Segment adulte, cirre invaginé

A ppareil femelle: Le vagin débouche antérieurement à la poche du cirre. Il se dirige d'abord vers le milieu du segment; là, il remonte vers l'avant en se dilatant. Il forme alors, dans la moitié antérieure du proglottis, un volumineux réceptacle séminal de $500 \mu$ 
sur $300 \mu$. Mais nous n'avons pas vu ce réceptacle à bords tendus ; les parois en sont toujours plissées et ridées. A la partie antérieure, ce réceptacle se rétrécit, forme une boucle, et le vagin descend par un canal assez large et sinueux jusqu'à l'ovaire. L'ovaire tétralobé s'étend à la partie postérieure du segment.

Les vitellogènes forment deux bandes latérales développées. Elles

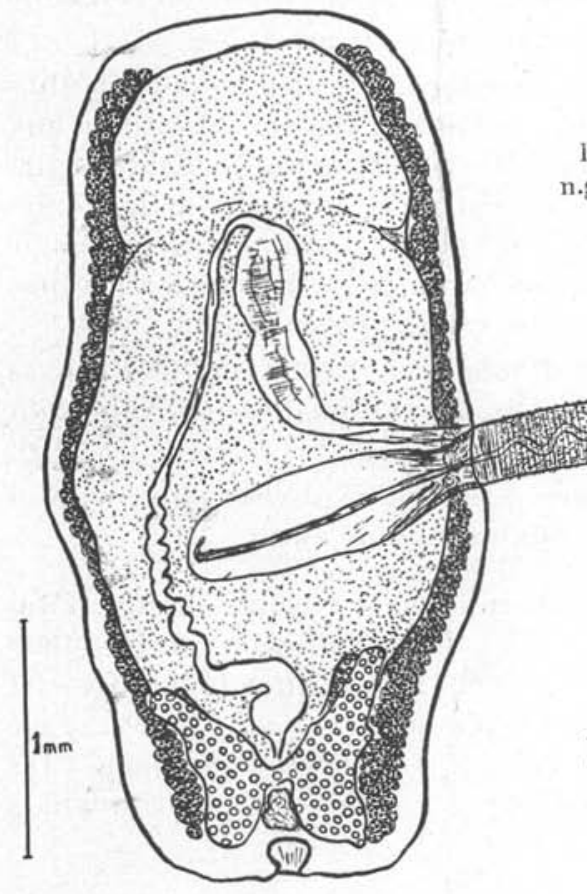

Fic. 7. - Rhabdotobcthrium dollfusi n.g.n.sp. Segment gravide, cirre évaginé

che du cirre, le vagin et une partie de l'ovaire.

empiètent légèrement et cachent dorsalement et ventralement les testicules.

L'utérus est sacciforme

7 (fig. 7) et, dans les proglottis détachés, occupe tout le segment, qui est alors un véritable sac à œufs, où seuls restent visibles la po-

Les œufs sont groupés par trois dans une coque sphérique de $80 \mu$ de diamètre. Ils contiennent un embryon hexacanthe de $15 \mu$.

Discussion : Ce Cestode, tel que nous venons de le décrire, ne peut entrer dans le genre Rhinebothrium Linton 1890 emended BAER 1948, car, dans son anatomie, il possède des testicules entre l'ovaire et la poche du cirre.

Il ne peut pas non plus entrer dans le genre Caulobothrium BAER 1948 , car il ne possède pas de «pédoncule céphalique net ». Il possède à la fois le caractère de l'un (scolex) et de l'autre (anatomie), avec cependant quelques structures particulières (vagin et pénis).

Notons cependant que nous avons décrit un Cestode, Rhinebo-

Ann. de Parasitologie, T. XXVIII, No 5-6. - 1953. 
thrium setiensis, parasite de Myliobatis aquila L., qui présente un vagin formant une boucle à la partie antérieure du segment. Mais ce ver ne possède pas de testicules entre la poche du cirre et lovaire du côté poral et nous l'avons, de ce fait, placé dans le genre Rhinebothrium.

"Le Cestode que nous avóns décrit ne pouvait entrer dans aucun des genres connus ; nous créons pour lui le genre Rhabdotobothrium

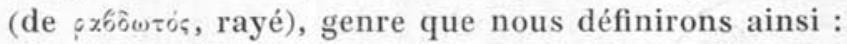

Strobile craspédote ; segments apolytiques. Scolex à quatre bothridies pédonculées à surface divisée en loculi. Pas de myzorhynchus. Pores sexuels marginaux alternant irrégulièrement. Testicules entre les glandes vitellogènes et l'ovaire. Un groupe de testicules entre l'ovaire et la poche du cirre du côté poral. Cirre épineux. Vagin débouchant antérieurement à la poche du cirre. Utérus sacciforme. Eufs pondus par groupes dans une enveloppe commune.

Nous nommons cette espèce Rhabdotobothrium dollfusi, en la dédiant à M. le Professeur R.-Ph. Dollfus. Rhabdotobothrium dollfusi devient l'espèce-type du genre.

La clé dichotomique des genres à bothridies divisées en loculi et à scolex sans myzorhynchus s'établira comme suit :

1. Pas de testicules entre la poche du cirre et l'ovaire du côté poral Rhinebothrium Linton 1890

Testicules entre la poche du cirre et l'ovaire du côté poral . . 2

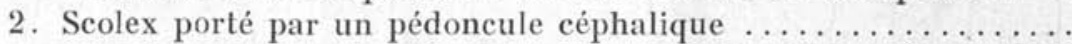

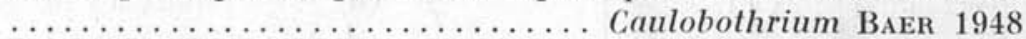
Scolex sans pédoncule céphalique .... Rhabdotobothrium n. g.

\section{RÉsumé}

Bothriocephalus tumidulus retrouvé est redécrit. On le place dans le genre Rhinebothrium Linton 1890 emend. BAER 1948. L'Echeneibothrium walga Shipley et Hornell 1906, dont on donne quelques précisions morphologiques et anatomiques, est, lui aussi, placé dans le genre Rhinebothrium. Ce Cestode, connu de Trygon walga, de l'Océan Indien, est signalé en Méditerranée et sur la côte française de l'Atlantique chez Dasyatis pastinaca. On décrit et étudie la position systématique de Rhabdotobothrium dollfusi n. g. n. sp. 


\section{BIBLIOGRAPHIE}

BaEr (J.-G.). - Contribution à l'étude des Cestoces de Sélaciens, I-IV. Bull. Soc. Neuchàt. Sc. Nat., 71, 1948, pp. 63-122, fig. 1-74.

Bresiser (J; G.). - Icones helminthum systema Rudolphi entozoologicum illustrantes, 18 planches, Vienne, 1824 .

Carus (S. V.). Prodomus Fanna Mediterranea sive descriptio Animalium maris Mediterranei incolarum. Part I, Cestodes, 1884, pp. 113-121.

Diesing (K. M.). - Revision der Cephalocotyleen. Abt. I Parnmecocotyleen. Sitzgob. d. l: Akad. Wiss. Wien. (Math. Natur. C1), 48, 1863, I.p. 200-345.

I)olffus (R.-Ph.). - Sur Acanthobothrium crassicolle K. WedL, 1855. Bull. Soc. Zool. France, 51, 1926, pp. 464-470, fig. 1-4.

Furrasin (O,). - Cestoüdea. Kükenthal's Handb. Zool., 2, 1931, pp. 141-416, fig. 176-435.

Joyeux et BaEr (J.-G.). - Cestodes. Faune de France, 1936, n" 30, pp. 1-613, fig. 1.569 .

Leuckant (F. S.). - Das Genus Bothriocephalus Rud. Zool. Bruchs., I-III, 1820.

I INTON (E.). - Notes on Entozoa of Marine Fishes of New-England, Part. 2, Cestodes. Rep. U.S. Fish. Comm. for 1887, 1890, pp. 718-899, pl. 1-15.

Notes on Cestode Parasites of Sharks and Skates. Proc. U. . Nat. Mus., $64,1924, f^{\circ} 2.511$, pp. 1-114, pl. 1-13.

LÉon-BonceA (L.). - Note préliminaire sur les Cestodes des Elasmobranches ou Sélaciens de la Mer Noire. Ann. Sc. Univ. Jassy, 19, 1934, pp. 345-369, fig. $1-10$.

Rudolphi (C. A.). - Entozoorum Synopsis cui accedunt mantissa duplex et indices locupletissimi, 1819, pp. 1-811, pl. I-III.

Shipley (A.) and Hornell (J.). - Cestode and Nematode parasites from marine fishes of Ceylon. Herdmann's Rep. on the Pearl Oyster Fisheries, Part. V, 1906, pp. 43-94, pl. 1-6.

Southwell (T.). - A monograph of the Tetraphyllidea with notes on related Cestodes. Mem. Liverpool 3c. Trop. Med., New series, 1925, n ${ }^{\circ}$ 2, pp. 1-368, fig. 1-243.

Vin Beneden (P.-J.). - Recherches sur la faune littorale de Belgique (Cestoïdes). Mém. Ac. Roy. Sc. Belg., XXV, 1850, pp. 1-200, pl. 1-24.

Mémoires sur les Vers intestinaux. Suppl. C.R.A.S. Paris, 1858, pp. 1-376, pl. 1-27.

Warde (R. A.) and McLeod (J. A.). - The Zoology of Tapeworms. Univ. of Minnes. Press., 1952, pp. 1-780, fig. 1-419.

(Station biologique de Sète) 